\title{
Retos para la docencia en el área de producción y operaciones en Colombia*
}

\author{
Juan Guillermo Ocampo Flórez" \\ Wilson Javier Castro Torres"
}

Recibido: 2 de febrero de 2016 • Revisado: 15 de febrero de 2016

Aprobado: 22 de febrero de 2016

\section{Resumen}

Varios autores han exaltado la importancia del perfil docente frente a la efectividad del proceso de enseñanza-aprendizaje y el desarrollo adecuado de las competencias en los estudiantes. Conscientes de esta realidad, las instituciones de educación superior han emprendido una carrera con el fin de identificar los elementos que inciden en la actividad docente e impactan de forma positiva o negativa la formación en las competencias de los estudiantes. Para algunos autores, el dominio del conocimiento es el soporte de las competencias que el docente pone en juego en el ejercicio de su tarea de formación, que se logra por la formación o la experiencia profesional que posee. Con estos elementos frecuentemente se construye el perfil apropiado para cada área de conocimiento y curso dentro de los programas de estudio.

Aunado a esto, en estudios sobre las tendencias para las competencias necesarias por el administrador, se requiere de un profesional con capacidad para la toma de decisiones bajo horizontes de incertidumbre, la optimización de recursos y la

" El artículo es resultado de la investigación desarrollada de forma conjunta por ASCOLFA, CESA y Universidad Piloto titulada: "Caracterización del perfil docente y sus competencias para la docencia”. El citado proyecto de investigación ha sido financiado conjuntamente por las citadas Academias.

* Magíster en Dirección Universitaria - Universidad de los Andes - Bogotá. Docente e investigador del Colegio de Estudios Superiores en Administración - CESA. Correo electrónico: jgocampo@cesa. edu.co.

*** Magíster en Administración de Proyectos - Universidad para la Cooperación Internacional; Magíster Profesional en Dirección y Admón. de Empresas (MBA)- Escuela Europea de Dirección y Empresa. Docente e Investigador de la Universidad Piloto de Colombia. Correo electrónico: wilsoncastro@unipiloto.edu.co. 
preservación y conservación del medio ambiente, las cuales se desarrollan en gran medida en los cursos que se tipifican en el área de Producción y Operaciones. Sin embargo, las escuelas de negocios en el país no han dado el paso que les permita apropiar esta tendencia, tanto por la baja incidencia de las asignaturas del área como por el desconocimiento del perfil del docente que las orienta.

El objetivo de las presentes líneas es identificar la pertinencia de la formación del perfil del docente de las asignaturas del área de Producción y Operaciones en los programas de Administración de Empresas de las Facultades de Administración afiliadas al capítulo Bogotá de ASCOLFA, para proponer acciones de fortalecimiento de una de las áreas de mayor potencial de la formación en gestión.

Palabras clave: Operaciones, competencias docentes, formación, perfil docente.

\title{
Challenges for teaching in the area of Production AND operations in Colombia
}

\begin{abstract}
Several authors have exalted the importance of teaching profile against the effectiveness of the teaching-learning process and proper development of skills in students. Aware of this reality the high education institutions have undertaken a career in order to identify elements that influence in the teaching and impact positively or negatively on skills training for students. For some authors, the domain of knowledge is supported by the teacher's skills put into play in the exercise of theirs formation's task, which is achieved by training or professional experience that he possesses. With these elements often it built the appropriate profile for each area of knowledge within the course curriculum.

It addition to this, studies on trends for the skills required by the administrator, requires a professional with capacity for take decision in under horizons of uncertainty, optimizing resources and the preservation and conservation of the environment, which is developed in the courses are classified in the area of Production and Operations. However, business schools in the country haven't taken the step that allows them to appropriate this trend, hence the low incidence of subjects and the lack of teacher guides that profile in the area.
\end{abstract}


The purpose of these lines is to identify the relevance of the training of teachers of subjects in the area of Production and Operations Management programs of the Faculties of Business Administration affiliated chapter of ASCOLFA profile Bogotá, to propose actions to strengthen one of the areas of greatest potential for management training.

Keywords: Operations, teaching skills, training, teaching profile.

\section{Desafios PaRa o ENSINo Na ÁREA de PRODUÇão E OPERAÇões na Colômbia}

\section{Resumo}

Vários autores têm enaltecido a importância do perfil de ensino contra a eficácia do processo de ensino-aprendizagem e desenvolvimento adequado das habilidades nos alunos. Conscientes desta realidade, as instituições de ensino superior empreenderam uma carreira a fim de identificar os elementos que influem na atividade de ensino e impactam positivamente ou negativamente sobre a formação de habilidades dos alunos. Para alguns autores, o domínio do conhecimento é o apoio das habilidades que o professor põe em jogo no exercício da sua missão de formação, o que é conseguido através da formação ou experiência profissional que possui. Com estes elementos, muitas vezes se construí o perfil adequado para cada área de conhecimento e curso dentro do currículo de estudo.

Ligado a isto, em estudos sobre as tendências para as habilidades exigidas pelo administrador, é preciso de um profissional com capacidade para a tomada de decisões sob horizontes de incerteza, a optimização dos recursos e a preservação e conservação do meio ambiente, as quais se desenvolvem amplamente nos cursos que tipificam-se na área de Produção e Operações. No entanto, as escolas de negócios no país não deram o passo que lhes permite apropriar esta tendência, tanto pela baixa incidência das matérias da área como pela ignorância do perfil do professor que as orienta.

O objetivo destas linhas é identificar a relevância da formação do perfil do professor das matérias da área de produção e operações nos programas de Administração de Empresas das Faculdades de Administração inscritas a o Capítulo Bogotá de ASCOLFA, para propor ações para fortalecer uma das áreas de maior potencial da formação em gestão.

Palavras-chave: Operações, habilidades de ensino, formação, perfil de ensino. 


\section{Introducción}

Superar las falencias en los procesos de aprendizaje en la formación en gestión incorpora diversas variables, pero una de las que tiene mayor peso es el docente y sus competencias para la docencia. Caracterizarlos permite comprender su quehacer práctico e identificar aspectos esenciales que pueden afectar positiva o negativamente la formación ofrecida en los programas de Administración. El presente artículo pone énfasis en la formación en el área de Producción y Operaciones, considerado por muchos autores como el futuro del Administrador, partiendo de las megatendencias en tecnologías organizacionales que demandan la gestión del supply chain, business acumen y project management como pilar de las competencias del profesional (Schoenherr, Griffith, \& Chandra, 2014). Para dicha aproximación, se parte de la investigación realizada por ASCOLFA sobre la caracterización del docente de Administración de Empresas en su Capítulo Bogotá. El análisis toma como supuesto básico que la formación soporta el desarrollo de las competencias de un docente universitario (Schulman, 2013), ya que la docencia reproduce el conocimiento desarrollado en el área en que se ejerce, para lo cual se toma la formación superior de grado y posgrado en la misma área como requerimiento para buscar la correspondencia entre la formación ofrecida con la calidad del saber propio que se enseña; y el dominio de la temática incide directamente en las competencias para el diseño, desarrollo y evaluación del curso. Aunque la formación no es la única variable de incidencia, metodológicamente se aíslan las demás variables, para abrir la discusión en torno al tema, ya que el análisis que se realiza parte de la pertinencia del perfil del docente desde su formación profesional y posgraduada, con la cual se trabajó en la investigación inicial, y tomando como muestra 48 profesores que declaran orientar asignaturas en el área señalada.

\section{Revisión de literatura}

Algunos de los autores han definido las competencias del docente y realizan una mención específica para caracterizarlas. Tal es el caso de Perrenoud (2007) quien entiende la competencia docente como la práctica ejercida de una manera reflexiva, enfatizando que no es una acción técnica, sino que implica la comprensión del sentido de utilidad en la actuación en el ámbito ciudadano, lo que requiere la 
formación previa del docente como soporte para esta práctica crítica. Tardif (2004) incluye la experiencia cotidiana de trabajo y los saberes que se generan a partir de la misma como parte de la competencia docente.

De esta manera, la docencia se puede llegar a ejercer después de haber alcanzado la apropiación de dichos saberes por medio de la práctica de la profesión. Hernández (2005), por su parte, propone al docente como aquel que logra que el estudiante apropie el saber en el que forma, lo que implica el dominio del mismo para lograr su apropiación. En el caso de Zabalza (2003) la circunscribe a la práctica docente, que incluye el conocimiento profesional soportado por la formación en el área propia de conocimiento, la capacidad didáctica generada por formación en educación y la condición personal que lo habilita como formador. Schulman, a su vez, recupera la condición de efectividad de la práctica profesional que entrega la competencia y ve la docencia "relacionada con la enseñanza y comprende el desempeño observable de la diversidad de actos de enseñanza" (Schulman, Conocimiento y enseñanza: Fundamento de la nueva forma, 2005). Así, su eje es la enseñanza y los actos implementados para ejercerla, y la condición efectiva de la misma. Como se presenta, el dominio del conocimiento que el docente pone en juego en el aula de clase, soporta sus competencias. Y este dominio se puede alcanzar de dos maneras: por la formación profesional y por la experiencia profesional.

Afín a lo anterior, la investigación Caracterización del perfil del docente de los programas de Administración y sus competencias para la docencia que soporta este artículo (Ocampo, Castro, Becerra, \& Herrera, 2015), se asumió como definición de la competencia docente la capacidad didáctica del mismo, el diseño de actos formativos y el grado de apropiación del conocimiento que permite formar profesionales con capacidad de tomar decisiones en pro del desarrollo de la sociedad, sustentado en su formación pre y posgradual, la formación continuada en el área de conocimiento, las cuales son consideradas por los estudiantes participantes como necesarias para el ejercicio de una docencia de calidad. En este sentido, la regulación para la docencia en educación superior en Colombia pide acreditar los perfiles de los docentes, con formación igual o superior a la que se orienta, y de maestría o doctorado para los profesores de planta, también hace énfasis en relación y afinidad con las actividades académicas que desarrolla el docente (Ministerio de Educación Nacional, 2015), tomando como punto de partida la importancia del perfil docente en el desarrollo de las competencias del profesional. 


\section{Metodología}

La metodología de la investigación exploratoria a la que corresponde el trabajo que soporta este artículo, toma como base fuentes primarias a partir de una encuesta realizada a 295 docentes vinculados a más de 61 universidades ubicadas en Colombia, de los cuales 48 orientan alguna asignatura del área de Producción y Operaciones en los programas de Administración de las facultades afiliadas al Capítulo Bogotá de ASCOLFA. Esta encuesta se aplicó vía electrónica y física durante los meses de octubre, noviembre y diciembre de 2013. La misma es de carácter declarativo, en la cual se describen características relacionadas con competencias docentes formuladas por los teóricos en este campo y se incluye la formación recibida y los cursos orientados. La encuesta se realizó por invitación a las universidades, quienes convocaron a sus docentes de planta o cátedra, tiempo parcial o completo, con vinculación a la institución durante el período en el que se realizó el estudio.

Para evitar los sesgos, la invitación fue realizada a la totalidad de docentes y se tomó la información sin delimitar por núcleo de saber del conocimiento, el nivel de formación superior, las áreas del saber en que ejercen su docencia o la antigüedad de su vinculación con la institución. Para el análisis, se parte de la definición de las áreas de conocimiento de los planes de estudio, determinados por el derogado decreto 2566 de 2002 (Ministerio de Educación Nacional, 2003), que los denomina componentes de formación y a los cuales se vinculan las asignaturas que orientan los docentes, relacionando sus títulos de formación de grado o posgrado y educación no formal, categorizados con los mismos componentes del decreto en mención.

Para identificar los cursos del área de Producción y Operaciones, se tomaron los planes de estudio de los programas de Administración que participaron en la investigación, incluyendo las 17 instituciones de educación superior en que los 48 docentes de la muestra se encuentran y se solicitó a un grupo de expertos su opinión sobre los cursos pertenecientes al área, la agrupación temática en que se subdividen y su percepción sobre el aporte que puede dar un docente con la formación de grado y posgrado a ese curso en particular. El análisis para la pertinencia de los títulos obtenidos por los profesores se realiza por mapas de proximidad, se tomó esta percepción de los expertos que calificaron los títulos declarados por los profesores en cuatro categorías de acuerdo con el aporte que da el título recibido al curso orientado, tomando como supuesto que es la única variable de incidencia en el perfil, definido como "óptimo" cuando el título se encuentra directamente 
relacionado con el curso, "agrega valor" cuando existe una relación aunque no claramente directa, "ni agrega ni destruye valor" cuando la formación es en un área similar a la del pregrado pero sin relación con el curso, y "no agrega valor" cuando la formación es en un área diferente a la del pregrado y del área. Para hacer el análisis, se tomaron los resultados de los niveles de formación que son más valorados por los estudiantes en la investigación que soporta el presente artículo y que fue citada previamente.

\section{Resultados}

El resultado de la consulta de expertos permitió identificar cuáles de las asignaturas en los planes de estudio correspondían al área y cuáles eran las agrupaciones temáticas, que fueron definidas como Producción, Investigación de Operaciones, Logística, Procesos, Proyectos y Ambiental. El número de asignaturas encontradas se presenta en la siguiente tabla.

Tabla 1. Número de cursos de operaciones en planes de estudio

\begin{tabular}{ccc}
\hline Número de asignaturas & Número de IES & $\%$ \\
\hline 0 & 5 & $10,4 \%$ \\
\hline 1 & 2 & $4,2 \%$ \\
\hline 2 & 3 & $6,3 \%$ \\
\hline 3 & 17 & $35,4 \%$ \\
\hline 4 & 7 & $14,6 \%$ \\
\hline 5 & 7 & $14,6 \%$ \\
\hline 6 & 5 & $10,4 \%$ \\
\hline 7 & 1 & $2,1 \%$ \\
\hline 8 & 1 & $2,1 \%$ \\
\hline Total & 48 & $100 \%$ \\
\hline
\end{tabular}

Fuente: Elaboración propia

Como se observa, para el 50\% de la muestra, los planes de estudio tienen entre 3 y 4 cursos del área, mientras que en otras áreas que se denominan de soporte como la Cuantitativa, Inglés o Informática, pueden tener cinco o más cursos. En cuanto a la participación relativa, como se presenta en la siguiente tabla, el 63\% de los planes de estudio tiene menos del $6 \%$ de sus cursos dirigidos al área de Operaciones. Es 
de aclarar que los programas de Administración que no cuentan con asignaturas tipificadas dentro de esta área son algunas IES de las Fuerzas Armadas ofrecidas a sus oficiales. En primer lugar, se identifica que a pesar de la importancia del área en las tendencias sobre el perfil del egresado, para la muestra estudiada no se encuentra una orientación a formar en ella, al menos por la incidencia que los cursos ofrecidos tienen sobre la totalidad de los tomados por un estudiante en los planes de estudio vigentes.

En la siguiente tabla, se presentan las frecuencias cumuladas, en la que se ratifica el bajo impacto que los cursos del área tienen sobre los planes de estudio, ya que para el 62,5\% de las instituciones que hacen parte del estudio, tienen una participación en ella del $6 \%$ o menos.

Tabla 2. Participación relativa de los cursos de operaciones en planes de estudio

\begin{tabular}{cccc}
\hline Rango & \# de IES & $\%$ & \% Acumulado \\
\hline No aplica & 4 & $8,3 \%$ & $8,3 \%$ \\
\hline $0 \%$ & 1 & $2,1 \%$ & $10,4 \%$ \\
\hline $2 \%$ & 2 & $4,2 \%$ & $14,6 \%$ \\
\hline $4 \%$ & 4 & $8,3 \%$ & $22,9 \%$ \\
\hline $5 \%$ & 7 & $14,6 \%$ & $37,5 \%$ \\
\hline $7 \%$ & 12 & $25,0 \%$ & $62,5 \%$ \\
\hline $8 \%$ & 5 & $10,4 \%$ & $72,9 \%$ \\
\hline $9 \%$ & 2 & $4,2 \%$ & $77,1 \%$ \\
\hline $10 \%$ & 5 & $10,4 \%$ & $87,5 \%$ \\
\hline $11 \%$ & 2 & $4,2 \%$ & $91,7 \%$ \\
\hline $12 \%$ & 1 & $2,1 \%$ & $93,8 \%$ \\
\hline $19 \%$ & 2 & $4,2 \%$ & $97,9 \%$ \\
\hline Total & 1 & $2,1 \%$ & $100,0 \%$ \\
\hline & 48 & $100 \%$ & \\
\hline
\end{tabular}

Fuente: Elaboración propia

Así, se observa que existe una estructura asimétrica en la mayoría de planes de estudio, en los que Operaciones presenta una baja participación en el grupo de asignaturas, en contradicción con las tendencias internacionales ya mencionadas. 
Por otro lado, en la caracterización general de los docentes que declaran orientar cursos en el área, se encuentra que el 64,6\% son del sexo masculino y el $78,5 \%$ tiene por encima de 35 años. En cuanto a su vinculación laboral, el 47,9\% tiene contrato como profesor de hora cátedra, el 6,3\% de medio tiempo y el 45,8\% como tiempo completo aunque el 56,3\% tiene una vinculación igual o inferior al semestre, el 39,6\% entre 10 y 12 meses y solo el 4,2\% cuenta con contrato a término indefinido. En cuanto a su remuneración, el 50\% devenga menos de 2 millones de pesos colombianos, y el 29,2\% entre 2 y 3 millones. Las actividades adicionales a la docencia que incluye su contrato alcanzan el 29,2\% para la investigación, 20,8\% para la extensión, 25\% para la asesoría de trabajos de grado y el 39,6\% para las actividades administrativas en su facultad. En lo referente a la experiencia docente, el 29,2\% tiene menos de cinco años de experiencia en docencia, incluyendo el $16,7 \%$ que cuenta con menos de dos años; mientras que solo el 25\% tiene más de 15 años ejerciendo esta profesión y el 50\% lleva menos de tres años de vinculación con la IES actual.

Entre las actividades adicionales a la docencia, las más frecuentes son la publicación de artículos en revista de divulgación nacional con el 35\% de los profesores, la producción de material de enseñanza con el $33 \%$, el capítulo de libro con $17 \%$ y los casos de enseñanza con 15\%. Igualmente, el $48 \%$ declara estar estudiando, el $42 \%$ realizar trabajos de consultoría como independiente o con una firma, el 19\% ejercer como profesional independiente, el 18\% tener una empresa como comerciante, pequeño industrial o artesano, y el 21\% declara estar buscando un empleo más estable, de los cuales el 17\% dejaría la docencia en el caso de encontrarlo.

Al relacionar el número de cursos y las áreas a las que pertenecen, se encuentra en primer lugar que el $4 \%$ orienta una única asignatura, el $15 \%$ dos, el $25 \%$ tres y el $19 \%$ cuatro, mientras que el $37 \%$ orienta cinco o más, incluyendo $12 \%$ que orientan más de diez en otras cuatro a seis IES, con dedicaciones superiores a 20 horas directas de clase en la primera y otras 20 horas o más en las demás. Por esta misma razón, orientan cursos en las áreas de Formación Básica, Administración y Organizaciones, Economía y Finanzas, Mercadeo, Gerencia de Personal, Informática, Formación Socio-Humanista y Operaciones.

Cuando se solicitó que declararan las asignaturas que orientaban, pregunta no inducida que permite que el profesor jerarquice el orden de acuerdo con sus motivaciones individuales, se encuentra el siguiente resultado: 


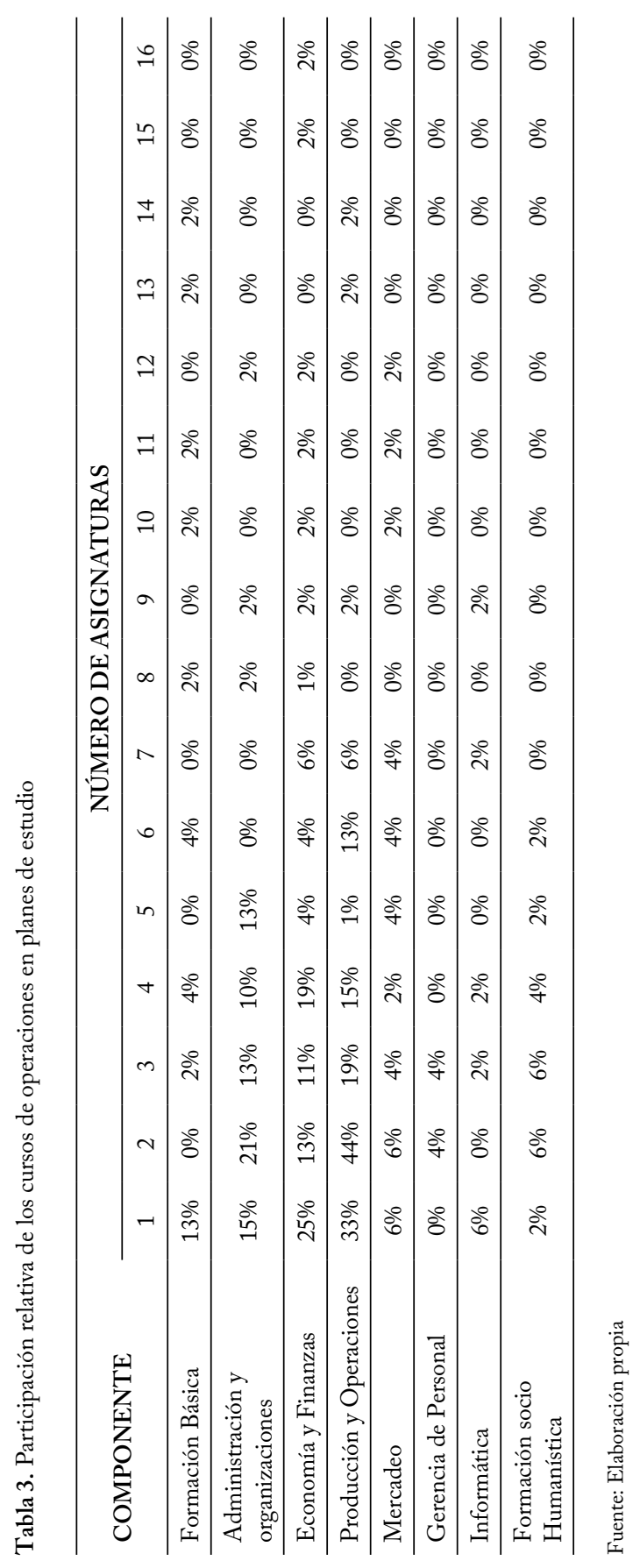


Los resultados permiten reflexionar sobre el perfil del docente y la pertinencia temática del ejercicio de la docencia que desarrollan. Docentes que acompañan áreas tan disímiles y que orientan tal cantidad de cursos, llevan a preguntarse por la calidad de la docencia que ejercen. Pero, desde el punto de vista de las necesidades particulares del profesor, pueden encontrar explicación en la necesidad de mejorar sus ingresos, acompañando cursos de otras áreas o incluyendo en su portafolio como docente cursos de Producción y Operaciones, aunque no tenga la formación para hacerlo con el dominio temático que se requiere.

$\mathrm{Al}$ profundizar en el análisis de la formación de estos profesores sin tener en cuenta el área de la misma, la especialización alcanza el 77\%, la maestría el 39,6\% y no se encontró a ningún docente con formación doctoral, siendo los dos primeros ligeramente superiores al promedio encontrado para los docentes de todas las áreas. Al identificar los docentes por cada una de las agrupaciones temáticas del área de Producción y Operaciones, el nivel de pertinencia de los estudios de grado y posgrado de los docentes es disímil, ya que se encuentran títulos pertenecientes a otros componentes que no tendrían, en un primer nivel de análisis, relación con el área en cuestión. Se encuentran por debajo de los promedios generales la especialización en las agrupaciones de producción e investigación de operaciones; en maestrías para ésta última y logística; y en doctorado para todas las áreas, tal como se observa en la Tabla 4.

Tabla 4. Formación académica de los docentes

\begin{tabular}{lcccc}
\hline Areas temáticas & Segundo pregrado & Especialización & Maestría & Diplomado \\
\hline Producción & $14,3 \%$ & $57,1 \%$ & $42,9 \%$ & $78,6 \%$ \\
\hline Operaciones & $14,0 \%$ & $43,0 \%$ & $29,0 \%$ & $100,0 \%$ \\
\hline Logística & $0,0 \%$ & $83,0 \%$ & $0,0 \%$ & $83,0 \%$ \\
\hline Procesos & $10,0 \%$ & $80,0 \%$ & $60,0 \%$ & $50,0 \%$ \\
\hline Proyectos & $7,0 \%$ & $79,0 \%$ & $50,0 \%$ & $71,0 \%$ \\
\hline Ambiental & $6,3 \%$ & $77,1 \%$ & $41,7 \%$ & $70,8 \%$ \\
\hline
\end{tabular}

Fuente: Elaboración propia

Además de estos aspectos, como se presenta en la Tabla 5, se destaca que la formación que poseen los docentes no necesariamente tiene un nivel de afinidad o pertinencia con las asignaturas que orientan. De acuerdo con el juicio de expertos, los docentes que orientan asignaturas dentro de la agrupación producción presentan 
un grado de pertinencia del 50\% en su pregrado, 33\% a nivel de especialización, $50 \%$ maestría y solo $18 \%$ en formación continuada. Para la agrupación investigación de operaciones alcanza los niveles de pertinencia del 38\% en pregrado, 25\% en especialización, $50 \%$ maestría y $42 \%$ en formación continuada. En logística, la afinidad del pregrado llega al 50\%, en especialización 88\%, sin docentes con formación a nivel de maestría y $18 \%$ en educación continuada. En procesos es $72 \%$ la pertinencia en pregrado, 45\% en especialización, 50\% maestría y 22\% diplomados. En la agrupación de proyectos $60 \%$ pregrado, $47 \%$ especialización, $86 \%$ maestría $21 \%$ diplomado. Para cerrar este aspecto en la categoría ambiental la pertinencia del pregrado es del 50\%, especialización 34\% a nivel de maestría 66\% y formación continuada es nula. Es decir el nivel de pertinencia de su formación de acuerdo con la asignatura que orientan se muestra débil, tal como se resume en la tabla 5.

Tabla 5. Niveles de pertenencia en la formación

\begin{tabular}{lcccc}
\hline Área temática & Pregrado & Especialización & Maestría & Diplomado \\
\hline Producción & $50 \%$ & $33 \%$ & $50 \%$ & $18 \%$ \\
\hline Operaciones & $38 \%$ & $25 \%$ & $50 \%$ & $42 \%$ \\
\hline Logística & $50 \%$ & $88 \%$ & $\mathrm{n} / \mathrm{a}$ & $28 \%$ \\
\hline Procesos & $72 \%$ & $45 \%$ & $50 \%$ & $22 \%$ \\
\hline Proyectos & $60 \%$ & $47 \%$ & $86 \%$ & $21 \%$ \\
\hline Ambiental & $50 \%$ & $34 \%$ & $66 \%$ & $0 \%$ \\
\hline
\end{tabular}

Fuente: Elaboración propia

Si la formación en el área de conocimiento en que se orientan los cursos es determinante para el perfil del docente, los resultados encontrados muestran que en el mejor de los casos el perfil más apropiado se encuentra en la agrupación temática de proyectos y procesos, seguido de logística, ambiental, investigación de operaciones y en último lugar se encuentra producción. Pero se puede identificar que para el área se tienen gaps importantes en el perfil de sus docentes, en particular cuando en la investigación se encontró que la formación del grado es determinante de la calidad de la docencia y para ella el máximo nivel de pertinencia cubre menos de una cuarta parte de los docentes para el caso de procesos, pero escasamente llega al 38\% en el caso de investigación de operaciones. Y en el caso de los posgrados, aunque se presentan niveles aceptables de estudios previos en logística y proyectos 
para especializaciones y maestrías, las restantes se encuentran por debajo del $47 \%$ en el caso de las primeras y de $66 \%$ en el caso de las segundas. Mientras que el diplomado como opción de actualización, en ninguno de los casos presenta un impacto importante para el perfil de los docentes del área, al menos para el caso de la muestra estudiada.

\section{Conclusiones}

Frente a la capacidad que tienen las facultades de Administración para dar respuesta a las nuevas tendencias y requerimientos del mercado, cabe el cuestionamiento si se está incluyendo el área de Producción y Operaciones como una de las prioridades para el administrador que requiere el mercado en los próximos años, ya que este exige la capacidad de entender el mundo de los negocios con una visión más allá de las fronteras, lo que representa mayores retos en temas relacionados con esta área. Incluir el 6\% o menos de asignaturas, dentro de los planes de estudio orientadas hacia el fortalecimiento de las competencias para gerenciar desde la cadena de abastecimiento global, permite afirmar que los egresados siguen saliendo con la mirada de empresas de desarrollo local, que tienen cada vez menor peso en la economía del país en cuanto a generación de desarrollo económico para el mismo y para los profesionales que a ellas se vinculan.

Y como consecuencia de lo anterior, la importancia que se está dando a la selección de profesores que orienten las asignaturas del área no presenta la relevancia que debería tener. Un reflejo de ello son el número de asignaturas en diferentes áreas que deben orientar, el tipo de contratación laboral del docente y su duración, que llevan a que uno de cada cinco docentes estén buscando otro trabajo y casi nueve de cada diez de estos docentes está dispuesto a dejar la docencia por otro empleo. Se podría deducir que hay un porcentaje significativo de docentes que la ejercen de manera transitoria, o mientras termina estudios posgraduales o mientras logran una "mejor" ubicación en la que encuentren condiciones salariales y de motivación superiores.

Pero al analizar el perfil del docente bajo la óptica de sus estudios de grado y posgrado como soporte para las competencias para ejercer su tarea con la calidad esperada, el resultado es más débil. Aunque en el número de docentes con estudios de especialización y maestría se encuentran un poco por encima del promedio de todas las áreas, el nivel de pertinencia entendido como la correspondencia del área en el que se formó y los cursos que orientan presentan resultados débiles, lo que 
lleva a cuestionar la calidad de la orientación ofrecida a sus estudiantes, en especial cuando tienen a su cargo cursos de varias áreas.

Relacionado con lo planteado más arriba, las bajas condiciones reales de su vinculación laboral lleva a que docentes con estudios previos que no se ajustan a los requerimientos del área estén acompañando cursos en un área crítica para el desempeño futuro del administrador. La baja afinidad de su formación con la materia que orientan, que alcanza un nivel de pertinencia en la formación a nivel de pregrado débil, en el que se destaca el 38\% de afinidad en la agrupación temática de operaciones y un máximo de un $60 \%$ de pertinencia en la de proyectos; en el nivel de especialización, el área temática de ambiental solo alcanza el 34\%; mientras que en educación continuada la pertinencia para todas estas agrupaciones tiene un impacto relativo bajo. Esto lleva a pensar que se requiere elevar el nivel de exigencia en el cumplimiento del perfil docente, de acuerdo con la formación afín al área temática en que se forma dentro del componente de Producción y Operaciones, bajo el supuesto que ésta es determinante para la plena exposición de las competencias docentes tanto en el diseño del curso, como en su desarrollo y el proceso de evaluación. Porque no es posible, desde la posición conceptual ya presentada, pensar en un docente que pone en plena operación sus competencias, cuando no tiene el dominio requerido para que su ejercicio sea de alta calidad.

Otro elemento a tener en cuenta es la oferta de programas posgraduales específicos en el área de Producción y Operaciones, que no siempre corresponde con los requerimientos del área, en particular desde los títulos ofertados. En un proceso regulado por la demanda, cabe la pregunta si el número de profesionales, entre ellos los docentes, que buscan continuar con su formación en estos campos específicos de conocimiento no son suficientes para mantener un programa de posgrado. Tal es el caso de la agrupación temática logística, en el que la oferta de programas a nivel de maestría en la ciudad de Bogotá solo alcanza tres, en operaciones que es de cinco y en supply chain ninguno, siendo éstos campos en los que profesionales con altas competencias son requeridos por las organizaciones empresariales de hoy y, especialmente, las del futuro. La oferta de programas como estos debería fortalecerse a fin de conseguir tanto un perfil pertinente de los docentes como para alcanzar la formación requerida en los profesionales de la administración.

Finalmente, las competencias del perfil del docente del área de Producción y Operaciones aún no está dando respuesta a la oportunidad que tienen las escuelas de negocios de generar una diferenciación en sus estudiantes de administración, con 
la clara orientación hacia la pertinencia que dé respuestas a un mundo globalizado que exige profesionales formados para gerenciar empresas y tomar decisiones que impacten de manera positiva su acciones en operaciones de comercio, producción, distribución, logística y proveeduría en la región pero con una incidencia en el mercado global.

\section{Referencias}

Hernández, C. A. (2005). Colombia aprende. Recuperado de http://www.colombiaaprende. edu.co/html/mediateca/1607/articles-128237_archivo.pdf

Ministerio de Educación Nacional. (2003). Ministerio de Educación Nacional. Recuperado de http://www.mineducacion.gov.co/1621/articles-86425_Archivo_pdf.pdf

Ministerio de Educación Nacional. (2015). Decreto 1075 del 26 de mayo de 2015. Bogotá: Imprenta nacional.

Ocampo, J., Castro, W., Becerra, G., y Herrera, B. (2015). Caracterización del perfil del docente de los programas de Administración y sus competencias para la docencia. Bogotá: ASCOLFA.

Perrenoud, P. (2007). Diez nuevas competencias para enseñar. Recuperado de http://newz33preescolar.files.wordpress.com/2012/05/res+C7umen-10-competencias.pdf

Schoenherr, T., Griffith, D., \& Chandra, A. (2014). Intangible capital, knowledge and new product development competence in supply chains: process, interaction and contingency effects among SMEs. . International journal of production research, vol. 56, 4916-4929.

Schulman, L. (2005). Conocimiento y enseñanza: Fundamento de la nueva forma. Curricullum y formación del profesorado, $\mathrm{Vol} 9, \mathrm{~N}^{\circ} 2$.

Schulman, L. (2013). Those Who Understand: Knowledge Growth in Teaching. Journal of Education, Vol 183, 1-11.

Tardif, M. (2004). Los saberes del docente y su desarrollo profesional. Madrid: Narcea.

Zabalza, M. A. (2003). Competencias docentes del profesor universitario: calidad y desarrollo profesional. Madrid: Narcea. 